\title{
RELATIONSHIP OF CORONAL MASS EJECTION EVENTS WITH SOLAR FLARES AND CORONAL HOLES
}

\author{
V K Verma \\ U.P. STATE OBSERVATORY, NAINI TAL - 263 129, INDIA
}

\begin{abstract}
We present an analysis of the relationship between coronal mass ejection (CME) events with solar flares and coronal holes. Out of $79 \mathrm{CME}$ events whose location and span are known, $10 \mathrm{CME}$ event circles contain solar flares. On the other hand out of $71 \mathrm{CME}$ events (selected for the study with coronal holes) $40 \mathrm{CME}$ event circles contain coronal holes. We have examined the above associations by calculating the Yule's coefficients of association and found that the CME events and solar flares are statistically not associated while CME events and coronal holes are statistically associated. This does not necessarily indicate that CMEs come from coronal holes as claimed by Hewish and Bravo (1986).
\end{abstract}

\section{OBSERVATIONAL DATA, ANALYSIS, RESULTS AND DISCUSSIONS}

To study the relationship of the CME's with the solar flares and coronal holes, the CME data has been taken from a paper by Sheeley et al (1984). The solar flares related to CME's observed during 1979-1982 were identified by Sheeley et al (1984) and by Robinson et al (1986). The coronal hole locations (10830 A) for the above period are taken from a paper by Stewart et al (1985).

For elucidating the study of the temporal and spatial relation between CMEs and solar flares, and CMEs and coronal holes, we have drawn a circle of radius equal to the span of CMEs, say \pm 10 , (i.e. a circle of radius 10 degrees) location in latitude say S25, (i.e. 25 degree south of the solar equator). If the CME circle drawn above contains a solar flare or coronal hole we infer that the CME event and coronal hole or solar flare are related to each other. In Figure 1 we have shown a synoptic map of the Sun for the period May 23 - June

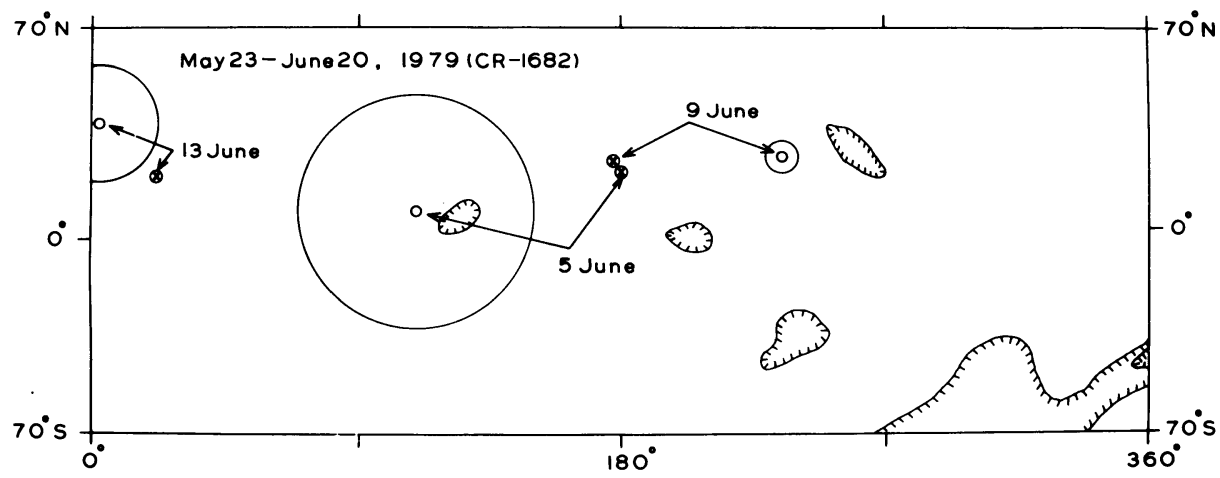

Fig. 1. Synoptic map, showing locations of CME events of 5th, 9th and 13th June 1979. 450

E. R. Priest and V. Krishan (eds.), Basic Plasma Processes on the Sun, 450-451.

(C) 1990 IAU. Printed in the Netherlands. 
20, 1979 (CR-1682). In Figure 1 we have shown CME circles for 3 events viz. 5 June 1979, 9 June 1979 and 13 June 1979. As it is clear from Figure 1 that 5th June 1979 CME circle contains a coronal hole while the flare temporarily associated with this CME as identified by Sheeley et al (1984) and by Robinson et al (1986) is located outside the CME circle. The location of the associated flares is shown in Figure 1 by a cross mark inside a small circle. From Figure 1 it is clear that the 5th June 1979 CME event is associated with the coronal hole and associated with the solar flare. The 9th and 13th June 1979 CMEs are associated neither with coronal holes nor solar flares. Several figures similar to Figure 1 are drawn to carry out the analysis.

Out of 79 CMEs whose location and span are known $10 \mathrm{CME}$ event circles contain solar flares. On the other hand out of $71 \mathrm{CMEs}$ (selected for the study with coronal holes), 40 CMEs are found to be related with coronal holes. The detailed method for selecting events are described in a paper by Verma (1989). We have examined the above association by calculating the Yules coefficients of association and found that CMEs and solar flares are not statistically associated while CMEs and coronal holes are associated.

The overall aim of the present study is to understand better the origin of CMEs. Earlier in a detailed study of three interplanetary events Hewish et al (1985) found that mid-latitude coronal holes lay near the sources of the disturbances on the Sun and it was suggested that the eruption of a short-lived stream, or the acceleration of an existing stream from the hole, provided an explanation of the disturbances. CMEs originate from coronal holes. However, recent coronagraph observations from the Solar Maximum Mission have shown clearly that CMEs occur when pre-existing closed structures such as coronal arcades or helmet streamers lose equilibrium and erupt (Hundhausen et al, 1984; Low, 1986; Priest, 1988). The association with coronal holes can therefore be explained if the opening of the field during the CME creates a coronal hole or if it is the closed region to one side of a coronal hole that erupts.

\section{REFERENCES}

Hewish, A, Bravo, S : 1986, Solar Phys. 106, 185.

Hewish, A, Tappin, S J, Gapper, G R : 1985, Nature, 314, 137.

Hundhausen, A J et al in Solar Terrestrial Physics : Present and Future (ed D Butler and K Papadopoulos) NASA CP-2439, Chapter 6.

Low, B C : 1986, Highlights in Astron. 7, 243.

Priest, E R : 1988, Astrophys. J. 328, 848.

Robinson, R D et al : 1986, Solar Phys. 105, 239.

Sheeley, Jr N R et al : 1984, Astrophys. J. 279, 839.

Stewart, R T et al : 1985, Proc. Astron. Soc. Australia, 6, 231.

Verma, V K : 1989, Astron. Astrophys, to be submitted. 